\title{
Diseño y evaluación de un análisis predictivo en discrepancia oseodentaria maxilar superior en dentición mixta
}

\author{
Carlos López-Betancourt*, Esp. ${ }_{1}$, Daniela Rodríguez-Herrera, Odon. ${ }_{2}$, \\ Juan Gabriel Martínez, Odon.2
}

${ }_{1}$ Grupo de investigación en odontología GIOUMEB, Universidad Metropolitana de Barranquilla ${ }_{2}$ Universidad Metropolitana de Barranquilla

Recibido: 26 de mayo del 2016 Aprobado: 29 de agosto del 2016

*Autor de correspondencia: Carlos López B. Carrera 52 n. 82-39, Barranquilla, Atlántico, Colombia. Teléfono: 3153991897. Correo electrónico: cal678@hotmail.com

Cómo citar este artículo: López-Betancourt C, Rodríguez-Herrera D, Martínez JG. Diseño y evaluación de un análisis predictivo en discrepancia oseodentaria maxilar superior en dentición mixta. Rev Nac Odontol. 2017;13(25):35-41. doi: http://dx.doi.org/10.16925/od.v13i25.1706

Resumen. Introducción: el adecuado diagnóstico de una discrepancia dentoalveolar y un tratamiento temprano es clave para prevenir alteraciones oclusales y oseodentarias que complican los tratamientos futuros en denticiones permanentes. La presente investigación busca determinar la efectividad de las nuevas fórmulas simplificadas para el diseño y la evaluación del análisis predictivo en la discrepancia oseodentaria en dentición mixta, en niños de 7 a 10 años atendidos en la Fundación Hospital Universitario Metropolitano de Barranquilla. Metodología: estudio descriptivo correlacional en modelos de estudio de niños de 7 a 10 años que asistieron a la Fundación Hospital Universitario Metropolitano entre los años 2012 y 2013. El muestreo fue probabilístico aleatorio; luego de calcular el tamaño de la muestra con el 95\% de confianza y un margen de error del 5\%, se obtuvo una muestra de 119 niños. Se llevó a cabo una prueba piloto con el 27\% del total de la población, con el fin de realizar ajustes pertinentes. Resultados: las discrepancias obtenidas en los resultados de las fórmulas simplificadas arrojaron hallazgos significativamente similares a los valores obtenidos con Moyers, dando así un diagnóstico certero de las maloclusiones. Las medidas del tamaño de los caninos y las mediciones del espacio requerido para la erupción de los premolares fueron similares en cuanto a promedio y desviación estándar. Conclusión: las ecuaciones producto de esta investigación se usarán como herramienta para el análisis de predicción en dentición mixta en la población colombiana del Distrito de Barranquilla.

Palabras clave: análisis, dentición mixta, diagnóstico (DECS), fórmula. 


\title{
Design and assessment of a predictive analysis in upper jaw dento-osseous discrepancy in mixed dentition
}

\begin{abstract}
Introduction: Adequate diagnosis of dentoalveolar discrepancy and early treatment is key to prevent occlusal and dento-osseous alterations that complicate future treatments in permanent dentitions. The present research seeks to determine the effectiveness of new simplified formulas for the design and assessment of predictive analysis in mixed dentition dento-osseus discrepancy in children between 7 and 10 years old treated at the Hospital Universitario Metropolitano de Barranquilla. Method: Correlational descriptive study in study models of children between 7 and 10 years old who were treated at the Fundación Hospital Universitario Metropolitano between 2012 and 2013. Randomized probability sampling was used; after calculating the sample size with a $95 \%$ confidence interval and a margin of error of $5 \%$, a sample of 119 children was obtained. A pilot test was carried out with $27 \%$ of the total population in order to make relevant adjustments. Results: The discrepancies obtained in the results of simplified formulas yielded findings significantly similar to the values obtained by Moyers, thus providing an accurate diagnosis of malocclusions. The measurements of canine tooth size and the measurements of the space required for the eruption of premolars were similar in terms of mean and standard deviation. Conclusion: The equations resulting from this research will be used as a tool for the predictive analysis in mixed dentition in the Colombian population of Barranquilla.
\end{abstract}

Keywords: analysis, mixed dentition, diagnosis (DECS), formula.

\section{Elaboração e avaliação de uma análise preditiva em discrepância osseodentária maxilar superior em dentição mista}

Resumo. Introdução: o adequado diagnóstico de uma discrepância dentoalveolar e um tratamento precoce é fundamental para prevenir alterações oclusais e osseodentárias que complicam os tratamentos futuros em dentições permanentes. A presente pesquisa busca determinar a efetividade das novas fórmulas simplificadas para a elaboração e avaliação da análise preditiva na discrepância osseodentária em detenção mista em crianças de 7 a 10 anos atendidas na Fundação Hospital Universitário Metropolitano de Barranquila (Colômbia). Metodologia: estudo descritivo correlacional em modelos de estudo de crianças de 7 a 10 anos que estiveram na Fundação entre 2012 e 2013. A amostra foi probabilística aleatória; após calcular o tamanho da amostra com 95\% de confiança e uma margem de erro de 5\%, obteve-se uma amostra de 119 crianças. Realizou-se um teste-piloto com $27 \%$ do total da população a fim de realizar ajustes pertinentes. Resultados: as discrepâncias obtidas nos resultados das fórmulas simplificadas revelaram achados significativamente similares aos valores obtidos com Moyers, dando, assim, um diagnóstico exato das más oclusões. As medidas do tamanho dos caninos e as medições do espaço requerido para a erupção dos pré-molares foram similares quanto à média e ao desvio-padrão. Conclusão: as equações produto desta pesquisa serão usadas como ferramenta para a análise de predição em dentição mista na população colombiana do distrito de Barranquilla.

Palavras-chave: análise, dentição mista, diagnóstico (DECS), fórmula. 


\section{Introducción}

En el desarrollo normal del individuo, intervienen factores como la combinación racial, cambios en los patrones alimenticios, variabilidad individual, factores ambientales, factores hereditarios y desarmonías miofuncionales (hábitos), entre otros. Esto lleva al desarrollo de maloclusiones y discrepancias dentolabiales que con el tiempo se han ido dilucidando, formando así un acopio de conocimiento que ha permitido predecirlas hoy en día en etapas tempranas e interceptarlas para evitar su expresión total en la adolescencia [1-3]. Otros procesos como la alimentación y la nutrición, que están influenciados por aspectos biológicos, ambientales y socioculturales, durante la infancia juegan un importante papel en un buen desarrollo [4].

La oclusión se define como normal en un sistema biológico y fisiológico cuando hay ausencia de patología y cuando el aparato masticatorio tiene la capacidad de adaptarse a desviaciones mínimas dentro de un margen de tolerancia. Siendo así, en la oclusión se contemplan, además de la relación e interdigitación de los dientes, las relaciones entre estos y los tejidos que los rodean (duros y blandos) [5].

Desequilibrios como las anomalías transversales se presentan con frecuencia a edades tempranas y son causados por problemas funcionales [6]. Una vez eliminados los desequilibrios derivados de los malos hábitos (como deglución infantil, succión de dedo y chupete, interposición de labio y respiración bucal), es imprescindible reeducar la neuromusculatura [7].

La maloclusión más frecuente en la dentición mixta y permanente es la discrepancia, es decir, la desarmonía entre el tamaño de los dientes y de los maxilares. El momento ideal para intervenir es en la dentición mixta temprana, pues los cambios que influyen en las dimensiones de los arcos se dan en esa edad [8]. Los arcos pueden desarrollarse normalmente o afectarse por influencias genéticas y factores ambientales o funcionales [9].

El trauma dentoalveolar (TDA) está entre las lesiones que comprometen los dientes y sus estructuras periodontales [10]. La pérdida prematura de los caninos temporales tiene una mayor repercusión en el sector anterior, dado que son los últimos en hacer el recambio, garantizan el mantenimiento del espacio en el arco y favorecen una buena oclusión [11].
La desarmonía dentoalveolar es el factor predominante de las maloclusiones, y genera problemas de posición a nivel dentario como protrusiones, retrusiones, apiñamientosygiroversiones. Controlar y corregir estas alteraciones en proceso de desarrollo permitirá solucionar las anomalías de posición dentaria (por ejemplo, el apiñamiento dental, definido como la discrepancia que existe entre el tamaño del diente y el perímetro del arco); todo esto con el fin de preparar un mejor entorno orofacial a edad temprana, aprovechando el espacio libre diferencial entre dientes permanentes y deciduos [1-13]. El adecuado diagnóstico de una discrepancia dentoalveolar y un tratamiento temprano son claves para prevenir alteraciones oclusales que complican los tratamientos futuros en denticiones permanentes [13]. Un gran porcentaje de maloclusiones tienen su origen en el periodo de dentición mixta [2].

Un factor importante para el diagnóstico de discrepancia dentoalveolar es la predicción del tamaño mesiodistal de los caninos y premolares no erupcionados. Tanaka y Johnston [14] consideran que la precisión en la predicción puede ayudar a responder si el espacio disponible en el sector posterior es suficiente para la erupción de caninos y premolares permanentes, cuya mayoría estuvo basada en predicciones matemáticas mediante la utilización de ecuaciones de regresión lineal simple, radiografías y análisis estadísticos, teniendo en cuenta las correlaciones de los incisivos inferiores con otros grupos de dientes que han demostrado tener significativos valores predictivos. En el estadio de dentición mixta, no parece existir un efecto liberador del crecimiento cuando el tratamiento se instituye a esta edad [1].

La tabla de probabilidad de Moyers y la ecuación de Tanaka-Johnston son los análisis usados de manera rutinaria para este fin. Sin embargo, estos análisis fueron construidos con base en escolares de raza blanca de origen escandinavo y con base en el uso de la suma del tamaño de las coronas de los incisivos inferiores como factor predictor del tamaño de los dientes no erupcionados [14].

Diferentes estudios demuestran la falta de precisión en la predicción cuando estos análisis son realizados en poblaciones diferentes a la población base. Así mismo, estos estudios toman otros grupos de dientes como predictores del tamaño coronal de caninos y premolares no erupcionados y crean diferentes ecuaciones de regresión basadas en muestras 
de sus poblaciones para lograr mayor precisión en sus predicciones [1-14].

Entre los análisis de dentición mixta más notorios se destacan los estudios de Moyers [15], con sus tablas para superiores e inferiores, quizá las de mayor uso y difusión mundial, las cuales presentan varios niveles de probabilidad (de 5 a 95\%); sin embargo, Moyers aconseja utilizar el nivel de 75\%, porque lo encontró práctico desde el punto de vista clínico [12-15].

Los factores biológicos y ambientales asociados al impacto de las diversas costumbres socioculturales hacen que inevitablemente disminuya la exactitud teórica de la aplicación de los métodos de predicción de Moyers y Tanaka-Johnston en la población colombiana [12-17]. Algunos autores, en sus estudios individuales, comparan los métodos de predicción con los resultados reales en diferentes poblaciones para determinar si los distintos métodos funcionan y cuál de ellos es más preciso. Con este recorrido sobre el estado actual del conocimiento, se planteó el objetivo de diseñar y evaluar un método de análisis de discrepancia oseodentaria en dentición mixta, basado en la aplicación de fórmulas matemáticas, para la predicción del ancho mesiodistal de caninos y premolares superiores permanentes no erupcionados [14].

La finalidad de la investigación fue encontrar nuevas fórmulas (fórmula simplificada y alterna) para la predicción de tamaños mesiodistales de caninos y premolares permanentes no erupcionados, obtenida a partir de los modelos de estudio de una muestra de escolares de 7 a 10 años de la Fundación Hospital Universitario Metropolitano de Barranquilla.

\section{Materiales y métodos}

Estudio descriptivo correlacional en modelos de estudio de niños de 7 a 10 años que asistieron al Hospital Metropolitano de Barranquilla entre el 2012 y el 2013. Se calculó el tamaño de la muestra con el $95 \%$ de confianza y un margen de error del 5\%, y se obtuvo una muestra de 119 niños. Se hizo un proceso de calibración con la participación de un experto. El ejercicio fue realizado en diferentes momentos, se aplicaron tres calibraciones una vez a la semana, utilizando siempre el mismo sitio y los mismos instrumentos. Posteriormente, a cada uno de los cuatro observadores se le distribuyeron los diez modelos con el fin de que midiera el diámetro mesiodistal de los dientes mencionados y registrara lo encontrado en el formulario de recolección de información. Luego de revisado el formulario de recolección, se dio una calificación que arrojó una moderada fuerza de concordancia (Kappa 0,41). A los pacientes les tomaron modelos realizados por dos operadores, con técnica estandarizada usando un hidrocoloide irreversible y yeso piedra tipo III según indicaciones del fabricante.

Después, se aplicó primero una prueba piloto. A los pacientes se les entregó un formato de consentimiento para obtener el permiso de sus padres; y los niños que entregaron sus formatos de consentimiento firmados procedieron al diligenciamiento de una historia clínica. Los criterios de selección para el estudio fueron: pacientes en periodo de dentición mixta en cualquiera de sus tres etapas, que no tuvieran caries interproximales cavitacionales, que presentaran o no apiñamiento dental y que tuvieran o no malformaciones creadas o del desarrollo. Los pacientes que cumplieron los criterios de selección fueron incluidos en la muestra, y se les tomaron impresiones dentales que inmediatamente fueron lavadas con agua y vaciadas con yeso dental para ortodoncia.

La calibración de los observadores se realizó antes de iniciar la prueba piloto. El ancho mesiodistal de los dientes fue medido a partir de los modelos de estudio. Se midieron los dientes del lado derecho e izquierdo en un mismo paciente.

Se utilizó la prueba de Karl Pearson de correlación, que arrojó una correlación positiva alta para comparar el análisis de Moyers con la nueva fórmula simplificada. Se utilizó un formato de historia clínica y modelos de estudio (cubetas de impresión, alginato, yeso, lápiz, compás, regla milimetrada flexible) [17]. Los datos obtenidos fueron digitados en una planilla de Microsoft Excel 2013 y fueron exportados luego al paquete estadístico spss versión 19.0 en español.

\section{Aspectos éticos}

Se tuvieron en cuenta las consideraciones éticas establecidas en el tratado de Helsinky. Todo lo anterior se realizó bajo la supervisión y el aval del Comité de Bioética de la Universidad Metropolitana y según la Resolución 008430 de 1993 del Ministerio de Salud y Protección Social. 


\section{Resultados}

Las sumatorias de los incisivos superiores (centrales y laterales) y de los caninos superiores tanto derechos como izquierdos revelaron un promedio igual de 24,2 ( $\mathrm{DE} \pm 2,2)$. Así mismo, las sumatorias de los primeros premolares, segundos premolares y primeros molares superiores tanto derechos como izquierdos revelaron un promedio igual de 25,5 $(\mathrm{DE} \pm 2,2)$. No se encontró diferencia entre la sumatoria de incisivo central lateral y canino permanente y la sumatoria del primer premolar, segundo premolar y primer molar.

Tabla 1. Tamaño de los caninos en el diseño y evaluación de un análisis predictivo en la discrepancia oseodentaria en el maxilar superior para la dentición mixta fase II

\begin{tabular}{ccc}
\hline $\begin{array}{c}\text { Tamañocanino } \\
\text { ICS+ILS/2=C }\end{array}$ & Promedio & $\begin{array}{c}\text { Desviación } \\
\text { estándar }\end{array}$ \\
\hline Canino derecho & 8,0588 & 0,74269 \\
Canino izquierdo & 8,0735 & 0,74884 \\
\hline
\end{tabular}

Fuente: elaboración propia

Se evidenció que la sumatoria del espacio disponible para el derecho y el izquierdo reveló un promedio de 25,5 con $\mathrm{DE} \pm 2,0$, y para el espacio requerido la sumatoria de los derechos e izquierdos reveló un promedio de 23,3 . Se evidenció también que la discrepancia en la fórmula alterna arroja un resultado promedio de 2,18 con $\mathrm{DE} \pm 3,4$ para el derecho y de 1,97 con $\mathrm{DE} \pm 3,4$ para el izquierdo, en comparación con la fórmula de Moyers (tabla 2).

Tabla 2. Discrepancia entre la fórmula alterna y la fórmula de Moyers en el diseño y evaluación de un análisis predictivo en la discrepancia oseodentaria en el maxilar superior para la dentición mixta fase II

\begin{tabular}{|c|c|c|}
\hline $\begin{array}{l}\text { Discrepancia } \\
\text { fórmula alterna }\end{array}$ & Promedio & $\begin{array}{l}\text { Desviación } \\
\text { estándar }\end{array}$ \\
\hline Derecha & 2,1873 & 3,41971 \\
\hline Izquierda & 1,9773 & 3,56552 \\
\hline $\begin{array}{c}\text { Discrepancia } \\
\text { fórmula de Moyers }\end{array}$ & Promedio & $\begin{array}{c}\text { Desviación } \\
\text { estándar }\end{array}$ \\
\hline Derecha & 1,6378 & 2,13668 \\
\hline Izquierda & 1,4731 & 2,21086 \\
\hline
\end{tabular}

Fuente: elaboración propia
El coeficiente de correlación de las discrepancias derechas de estas dos distribuciones fue de $R=0,710$. El valor $p$ de significancia fue 0,000 $(\mathrm{p}<0,05)$, por lo que se rechaza la Ho, existiendo correlación entre las variables. Como el valor de $\mathrm{R}$ se encuentra entre 0,7 y 0,89 , la correlación entre las variables es positiva alta.

El coeficiente de correlación de las discrepancias izquierdas de estas dos distribuciones fue de $\mathrm{R}=0,753$. El valor $p$ de significancia fue 0,000 $(\mathrm{p}<0,05)$, por lo que se rechaza la Ho, existiendo también correlación entre las variables. Como el valor de $\mathrm{R}$ se encuentra entre 0,7 y 0,89 , la correlación entre las variables es positiva alta.

Se compararon los resultados de la nueva fórmula con los obtenidos con la fórmula de Moyers, y se halló que los resultados son aplicables en el 71\% y no aplicables en el $29 \%$.

\section{Discusión}

Los estudios realizados para el análisis de dentición mixta de Tanaka, Johnston y Moyers subestiman el valor real de los dientes permanentes. Sus resultados originales muestran que los incisivos centrales inferiores tienen una correlación con los caninos y premolares superiores e inferiores, lo cual concuerda con la mayoría de los estudios $[2,14]$. En cambio, los resultados arrojados en esta investigación muestran que los centrales laterales y caninos y el primer molar superior son los dientes seleccionados para la predicción del espacio requerido de los premolares, por lo cual se tiene más concordancia con los estudios realizados por Hixon et al. [13], en los que los dientes más opcionados para la predicción de espacios de premolares y caninos son centrales laterales y primer molar superior.

La fórmula simplificada utiliza las sumatorias de laterales y centrales divididas entre dos para predecir el diámetro mesiodistal del canino; una vez obtenido el diámetro del canino, se puede emplear la segunda fórmula en la que se suma el central lateral canino, el espacio de deriva que es 1,3 y se le resta el diámetro mesiodistal del primer molar superior para la predicción del diámetro mesiodistal de premolares superiores [16].

La nueva fórmula simplificada para la predicción de caninos y premolares podría ser más aplicable que la del análisis de Moyers, ya que el autor la realizó con base en un grupo étnico diferente. Las 
nuevas fórmulas simplificadas aportan gran ventaja, debido a que las mediciones se realizan directamente en los modelos de estudio previamente obtenidos de los pacientes, por lo cual la fórmula es sencilla, práctica y de fácil aplicación. Otras poblaciones que presenten criterios semejantes de selección realizados en este estudio podrían beneficiarse de esta fórmula $[3,17]$.

Teniendo en cuenta esto, se debe proponer una modificación de la fórmula según la selección del grupo a estudiar, ya que la variable étnica y la geográfica son de vital importancia tanto en el desarrollo de las maloclusiones como en el tratamiento que se les debe dar. Esta fórmula permite abordar con mayor precisión las anomalías en el desarrollo de la dentadura permanente, y la gran efectividad de un tratamiento ortopédico se basa en la atención temprana y eficaz de la anomalía, es decir que son factores importantes que facilitan la aplicación de la fórmula. Al arrojar, en la presente investigación, un resultado más real y más preciso de las medidas mesiodistales, se puede generar un tratamiento que actúe de manera más eficaz y que resuelva el problema en menor tiempo.

\section{Conclusión}

A partir de las 119 muestras obtenidas, se obtuvo que la predicción de diámetro mesiodistal de caninos reveló un promedio de 8,05 tanto para derecho como para izquierdo, con lo que demuestra que la diferencia de los diámetros de caninos derecho e izquierdo es mínima: canino derecho 8,0588, canino izquierdo 8,0735 , con una desviación estándar de 0,7. Las mediciones obtenidas del espacio requerido para la erupción de los premolares fueron similares tanto para el lado derecho como para el izquierdo, en cuanto al promedio y a la desviación estándar.

La nueva fórmula desarrollada en este estudio con base en los niños de 7 a 10 años que asistieron al Hospital Metropolitano demostró tener mayor eficacia para la predicción de los caninos y premolares permanentes superiores.

Las ecuaciones producto de estas investigaciones podrían usarse en la población colombiana y del Distrito de Barraquilla como herramienta para el análisis de predicción en dentición mixta.

\section{Agradecimientos}

Agradecemos a las doctoras Martha Rebolledo Cobos y Alexandra Marthe Manjarrés, quienes con dedicación y entrega asesoraron todo el proceso de la investigación.

\section{Referencias}

[1] Ramos PC, Adriazola ML, Evangelista A. Nueva ecuación de predicción de espacio requerido para dentición mixta basada en escolares de Lima Metropolitana. Rev Estomatol Herediana. 2011;21(2): 67-72.

[2] Botero PM, Cuesta DP, Agudelo S, Hincapié C, Ramírez C. Valoración de los métodos de análisis de dentición mixta de Moyers y Tanaka-Johnston en la predicción del diámetro mesiodistal de caninos y premolares no erupcionados. Rev Fac Odontol Univ Antioq. 2014;25(2):359-71.

[3] Nourallah AW, Gesch D, Khordaji MN, Splieth C. New regression equations for predicting the size of unerupted canines and premolars in a contemporary population. Angle Orthod. 2002;72(3):216-21.

[4] Macías AI, Gordillo LG, Camacho EJ. Hábitos alimentarios de niños en edad escolar y el papel de la educación para la salud. Rev Chil Nutr. 2012;39 (3):40-3. doi: http://dx.doi.org/10.4067/S0717-7518 2012000300006

[5] Romero MY, Bruno PD. Características clínicas y cefalométricas de la maloclusión clase iI. Odous Cientifica. 2013;14(1):37-45.

[6] Furlan FL, Lorean BMM. Un Tratamiento antiguo y vigente en ortodoncia: expansión rápida maxilar. Rev Soc Odontol Plata. 2015;25(51):5-14.

[7] Muller R, Piñeiro S. Los malos hábitos orales: rehabilitación neuromuscular y crecimiento facial. Revista Médica Clínica Las Conde. 2014;25(2):380-8.

[8] Vélez AL, Botero P. Tratamiento de paciente con discrepancia dentoalveolar. Revisión de literatura y presentación de un caso. Rev Ces Odont. 2005; 18(1):27-31.

[9] De Armas González Y, Alemán Estévez MG, Martínez Brito I, Hernández González YO, Almeida Bravo RJ. Diámetros mesiodistales coronarios de premolares superiores e inferiores en población con oclusión normal. Matanzas. Rev Med Electrón. 2014;36(5):540-50.

[10] Velásquez F, Mancialla C, Niño AY, Tirreau V, Cortés-Araya J, Rojas MC, et al. Patrones epidemiológicos del trauma dentoalveolar (patología ges) en pacientes adultos atendidos en un centro de trauma 
de Chile durante 2 períodos. Int J Odontostomat. 2014;8(2):191-9. doi: http://dx.doi.org/10.4067/S07 18-381X2014000200007

[11] Morera Pérez A, Ríos Paz Y, Villa Fernández DM, Cobas A. Presencia de anomalías dentomaxilofaciales en niños con pérdida prematura de caninos temporales. Medisur. 2016;14(3):334-7.

[12] De Paula S, Almeida MA, Lee PC. Prediction of mesiodistal diameter of unerupted lower canines and premolars using 45 degrees cephalometric radiography. Am J Orthod Dentofacial Orthop. 1995; 107(3):309-14. doi: http://doi.org/10.1016/S0889-5 406(95)70147-8

[13] Hixon HE, Oldfather RE. Estimation of the sizes of unerupted cusp and bicuspid teeth. Angle Orthod. 1958;28(4):236-40.
[14] Tanaka MM, Johnston LE. The prediction of the size of unerupted canines and premolars in a contemporary orthodontic population. J Am Dent Assoc. 1974;88(4):798-801. doi: https://doi.org/10.14219/ jada.archive.1974.0158

[15] Legovic M, Novosel A, Legovic A. Regression equations for determining mesiodistal crown diameters of canines and premolars. Angle Orthod. 2003;73(3): 314-8. doi: 10.1043/0003-3219(2003)073<0314:refd$\mathrm{mc}>2.0 . \mathrm{co} ; 2$

[16] Melgaco CA, Araujo MT, Ruellas AC. Applicability of three tooth size prediction methods for white Brazilians. Angle Orthod. 2006;76(4):644-9. doi: 10. 1043/0003-3219(2006)076[0644:aottsp]2.0.co;2

[17] Paredes V, Gandia JL, Cibrian R. A new, accurate and fast digital method to predict unerupted tooth size. Angle Orthod. 2006;76(1):14-9. doi: 10.1043/ 0003-3219(2006)076[0014:anaafd]2.0.co;2 